\title{
Der ärztliche Pestbegriff in historischer Sicht
}

\author{
Von Antoinette Stettler
}

\section{Die konstitutiven Elemente des Pestbegriffes und der Begriff der spezifischen Krankheit}

Die Bubonenpest, bei weitem die berüchtigtste Krankheit vergangener Jahrhunderte, ist gekennzeichnet durch außerordentlich hohe Tödlichkeit, sehr große Ansteckungskraft, hohes Fieber, Beulen (Bubonen) meist in der Leistenbeuge oder Achselhöhle, verschiedene Hautausschläge, Lungenentzündung, Nasenbluten u.a.m. Die Krankheit wird durch den Biß des Rattenflohs, der seinerseits von der pestkranken Ratte infiziert worden ist, auf den Menschen übertragen.

Im Jahre 1969 wird die Bubonenpest mit zwei Sätzen abgetan:

«Pest (Pasteurella pestis) verläuft als Bubonenpest, primär-septische Pest und Pestpneumonie. Bei allen drei Typen können die Pesterreger entweder aus dem Sputum, Punktat oder Blut nachgewiesen werden. ${ }^{1}$

Daß nur zwei Sätze gebraucht werden, liegt einmal daran, daß dank wirksamer Prophylaxe und Therapie die Zahl der Pestkranken seit der Mitte des 20. Jahrhunderts weltweit abgenommen hat. Ferner aber, und dies ist wichtiger in unserem Zusammenhang, zeigen diese zwei Sätze, daß die Diagnose der Bubonenpest offensichtlich kein prinzipielles Problem mehr bietet. Sie wird heute mit der in der Biologie üblichen an Sicherheit grenzenden Wahrscheinlichkeit, durch den Nachweis der Krankheitsursache, nämlich des Pestbakteriums, gestellt. Die Richtigkeit der Diagnose wird durch die Wirksamkeit der Therapie bestätigt.

Die Entdeckung des Pestbakteriums, neuerdings Yersinia pestis benannt, gelang dem Schweizer Alexandre Yersin und dem Japaner Shibasaburo Kitasato im Jahre 1894 anläßlich der Pestepidemie in Hongkong; den Übertragungsweg über den Rattenfloh entdeckten etwas später Georg Sticker et al. Mit dieser Entdeckung wurde das Ziel der Pathologie, wonach neben der Symptombeschreibung auch die Ursache und Entstehungsbedingungen einer Krankheit zu nennen seien, erreicht. Die Identität der Krankheit ist festgelegt und das je individuellen Variationen unterliegende Krankheitsgeschehen kann verbindlich, als dem Begriff «Bubonenpest» zugehörig, diagnostiziert werden. Seit 1894 kann man sagen, daß das seit langem «Pest» benannte Krankheitsbild eine spezifische Krankheit ist und einen spezifischen Erreger als Ursache hat.

An dieser Stelle ist ein kurzer Hinweis auf das Konzept der spezifischen Krankheit nötig. Man hat sich heute daran gewöhnt, für die Infektionskrankheiten und somit auch für die Pest den Begriff der spezifischen Krankheit zu verwenden, der 
sein Korrelat in der spezifischen Ursache bzw. im spezifischen Erreger hat. Diese Denkweise ist eine Errungenschaft der Bakteriologie des ausgehenden 19.Jahrhunderts, so daß es heute schwerfällt, sich überhaupt vorzustellen, daß man vordem nicht so dachte. Der Gedanke der spezifischen Krankheit begann sich im 17. Jahrhundert mit Thomas Sydenham zu kristallisieren, und zwar im Sinne der Krankheit als Spezies in Analogie zur botanischen und zoologischen Spezies. Was bei Sydenham vielleicht noch eher ein Gedankenspiel war ${ }^{2}$, wurde im 18. Jahrhundert von François Boissier de Sauvages und Carl von Linné wörtlich genommen, welche beide Werke hinterlassen haben, in denen die gesamten Krankheitserscheinungen in Ordnungen, Klassen, Gattungen und Spezies aufgeteilt sind. Im 19. Jahrhundert wurde der Gedanke von Johann Lucas Schönlein aufgenommen und traf in eine Zeit, wo von Seiten der Erforschung der Mikroorganismen ebenfalls spezifische pathogene Kleinstorganismen postuliert und alsbald auch gefunden wurden. Daraus resultierte der obenerwähnte Begriff der spezifischen Krankheit und des spezifischen Erregers.

Nach den genannten strengen Kriterien der Pestdefinition als spezifischer Krankheit erübrigt es sich beinahe, darauf hinzuweisen, daß eine retrospektive Pestdiagnose für die Zeit vor 1894 mangels der Möglichkeit eines retrospektiven Bakteriennachweises nicht gestellt werden kann. Es ist durchaus denkbar und wahrscheinlich, daß das historisch bloß aus der Symptomatologie zu belegende Krankheitsbild dem heutigen Pestbegriff nicht unterzuordnen wäre. Dasselbe gilt für alle Infektionskrankheiten, deren retrospektive Diagnose immer Ermessenssache bleibt. Die Lage ist für die retrospektive Bubonenpestdiagnose insofern günstiger, als die Pest das oben skizzierte recht typische Krankheitsbild bietet und bot, obwohl zahlreiche Pestbeschreibungen auch an Anthrax (Milzbrand) erinnern.

Mit der Einschränkung vor Augen, daß historische Diagnosen immer Ermessenssache sind, findet man denn wohl eine Beulenkrankheit unter den verschiedensten Namen in sehr zahlreichen Chroniken, Regesten, heiligen Büchern und ärztlichen Schriften erwähnt, so im Alten Testament ${ }^{3}$, im Corpus Hippocraticum ${ }^{4}$, bei Galen ${ }^{5}$, beim Kirchenvater Euagrius ${ }^{6}$, beim Historiker Procopius ${ }^{7}$, bei Avicenna, beim mittelalterlichen Arzt Gordonius, bis dann im Anschluß an das Massensterben von $1347 \mathrm{ff}$. und $1360 \mathrm{ff}$. eine wahre Flut von Monographien mit dem Titel «De peste» oder ähnlich das Auftreten der Bubonenpest glaubwürdig erscheinen lassen ${ }^{8}$. Aus allen diesen Hinweisen schien es der Medizinhistorie, namentlich des 19. Jahrhunderts, lohnend, die Geschichte der Pestzüge zu rekonstruieren. Es sei hier vor allem Georg Stickers unübertroffenes Standardwerk erwähnt ${ }^{9}$. 
Wenn es nun auch möglich ist, sich solche Seuchenzüge zu vergegenwärtigen, so heißt das auf keine Weise, daß mit dem jeweiligen Auftreten der «Pest» auch jedesmal ein klarer oder derselbe ärztliche Begriff der Pest als einer Krankheitseinheit, geschweige denn als einer spezifischen Krankheit bestanden habe, zumal zu postulieren ist, daß früher wie auch heute in Epidemiezeiten nicht nur eine einzige Infektionskrankheit vorkommt. Daß der Pestbegriff nicht immer scharf gefaßt wurde, hat seinen Grund auch darin, daß die Pest neben allen ihren sonstigen Eigenschaften eben auch eine sehr sporadische Krankheit ist. Das bedeutet, daß nicht alle Ärzte, die über die Pest zu schreiben sich verpflichtet fühlten, diese selbst miterlebt hatten. Für die Ärzte ergab sich ferner das zusätzliche Problem, die dann einmal beschriebene Pest in das überlieferte System der Krankheitslehre einzuordnen, was den Blick für die tatsächliche Eigenständigkeit der Pestkrankheit nicht unbedingt geschärft hat.

So waren es denn fast häufiger ärztliche Laien, welche die klareren Vorstellungen der Pest hatten; dennoch hingen vorwiegend von der ärztlichen Meinung zum Pestbegriff die Maßnahmen der Gesundheitspolitik, der Vorbeugung und der Therapie ab. Man steht also vor der etwas paradoxen Situation, daß zu gewissen Zeiten die maßgebenden Ärzte die unklareren, wenn auch komplexeren und, auf die Dauer der Entwicklung gesehen, dennoch sachgerechteren Vorstellungen der zu bekämpfenden Seuche hatten als die Laien.

\section{Die Konstituierung des ärztlichen Pestbegriffes}

\section{Pest und Tödlichkeit}

Die Hauptelemente des heutigen Pestbegriffes wurden oben absichtlich in der bestimmten Reihenfolge Tödlichkeit, Ansteckungskraft, Fieber, Bubonen, Lungenbefall, spezifisches Bakterium und pestkranke Ratte aufgezählt. Denn das ist zugleich die chronologische Folge, in der sie sich zum heutigen Pestbegriff konstituierten.

Das Element der Tödlichkeit gehört tatsächlich zum ältesten überlieferten Pestbegriff. Pest bedeutete zunächst nichts anderes als sehr tödliche, sehr verbreitete Krankheit, eine Bedeutung, die das Wort im übrigen auch noch heute im außermedizinischen Sprachgebrauch beibehalten hat. In diesem Sinne war die Pest des Thukydides ${ }^{10}$ eine Pest, auch wenn wir heute nicht mit Sicherheit sagen können, von welcher der heute eben als spezifisch aufgefaßten Infektionskrankheiten die Athener damals befallen waren.

«Mortalegga grande», «grande mortalité », «großes Sterben » wurde die Krankheitskatastrophe benannt, die $1347 \mathrm{ff}$. über Europa hereinbrach. Lazare Rivière 
(1640) sah in der Tödlichkeit das wesentliche Definitionselement der Pest ${ }^{11}$. Die Tödlichkeit ist zu allen Zeiten und auch heute noch als konstantes Element Bestandteil des Pestbegriffes.

\section{Pest, Ansteckungskraft und Pestzeit}

Ebenfalls schon seit der Antike gehört zum Pestbegriff die Ansteckungskraft, die contagio oder das contagium, und zwar so sehr, daß das Wort synonym zu Pest angewendet worden ist ${ }^{12}$. Die zahlreichen gleichzeitig auftretenden Krankheitsfälle legten die Vorstellung von der Ansteckungskraft nahe.

Der Ausdruck contagio bezeichnet den Sachverhalt, daß ein krankmachendes Agens, sei es als unsichtbares Tierchen, als Samen oder als Gift gedacht, irgendwie durch Berührung übertragen wird. Der Begriff der Ansteckungskraft nennt demnach auch die Entstehungsbedingungen der Pest: sie wird von der Außenwelt auf den Menschen übertragen, entsteht also nicht etwa in ihm selbst.

Wenn bis zum ausgehenden 19. Jahrhundert über die Entstehungsbedingungen der Pest Kontroversen geführt worden sind, betrafen diese nur in Ausnahmefällen die grundsätzliche Ansteckbarkeit der Pest. Sie galten vielmehr bloß der Art und Weise der Ansteckung und dem Wesen des krankmachenden Agens. Über diese beiden Punkte sind zum Teil aus der Beobachtung stammende, zum größeren Teil aber spekulative Ansichten gängig gewesen, die bis ins 17., zum Teil bis ins 19. Jahrhundert so oder ähnlich aussahen: Das Pestgift oder die Pesttierchen sind schon seit Anbeginn der Welt da oder entstehen in unbegrabenen Leichen, besonders nach einem Erdbeben, nach Mißernten und Hungersnöten. Das Gift oder die Tierchen werden durch direkten Kontakt (contagio im engeren Sinne) verbreitet oder in die Luft abgedunstet (miasma), vom Wind in die verschiedensten Gegenden geblasen und treffen auf den Menschen, den sie krank machen. Diese letztere, als Miasmalehre bekannte Ansicht hat heute ihren Nachklang in der Umgangssprache, wenn von angeblasenen Krankheiten oder von Luftverpestung die Rede ist. Folgerichtig wurde das Wetter auf allfällige Pestdünste hin beobachtet. Allgemein galten feuchte Luft als pestbringend sowie gewisse, angeblich die Wetterverhältnisse beeinflussende Konstellationen am Firmament.

Ferner waren vermehrt gesichtete Kröten, Schlangen sowie auch Mäuse und Ratten Anzeichen einer kommenden Pest. Man könnte darin ein Realwissen oder zum mindesten eine Ahnung um die Entstehungsbedingungen der Pest erblicken, denn, wie eingangs erwähnt, entsteht keine Bubonenpest ohne Rattenpest. Sticker ist dieser Frage nachgegangen und berichtet von Versuchen, die genann- 
ten Reptilien mit dem Pestbakterium zu infizieren, was aber offenbar nicht gelang ${ }^{13}$. Man ist daher eher geneigt, diese gesichteten Kriechtiere und Nager als retrograd imaginierten Ausdruck des Schreckens und Grauens vor der Pestzeit aufzufassen. Der tatsächliche Zusammenhang des Übertragungsweges von Ratten über Rattenfloh auf Mensch begann erst Ende des 19. Jahrhunderts klarzuwerden ${ }^{14}$.

Contagio, Miasma, Wetterverhältnisse, Konstellationen waren die Pestursachen innerhalb der Natur. Der Zorn Gottes als Pestursache war Ursache oberhalb der Natur, welche die natürlichen Ursachen nicht hinfällig machte, sondern ergänzte.

Diese hier kurz wiedergegebenen Entstehungsbedingungen der Pest wurden unter dem Oberbegriff Pestzeit bzw. Pestgegend zusammengefaßt. In dieser Form ist der Pestzeitbegriff heute ganz obsolet geworden. Doch stellt er nicht nur ein erschütterndes Raten über die wirklichen Pestursachen dar, sondern er bildete bis in die Zeit von Athanasius Kircher (1602-1680) hinein und darüber hinaus einen unabdingbaren Bestandteil der Pestdefinition.

Dabei gehen nicht alle Autoren so weit wie Laurent Joubert (1529-1583), der die Pest von der Luftverderbnis her definiert ${ }^{15}$. Aber immerhin war das Vorhandensein der Pestzeit für die Diagnose der Pest notwendiger als etwa die für uns pathognomonischen Bubonen, dergestalt, daß ein Kranker die Pest hatte, wenn ein akut fiebriges Kranksein festgestellt wurde und gleichzeitig Pestzeit herrschte.

\section{Pest und Fieberlehre}

Auch das hohe, mit raschem Kräftezerfall einhergehende Fieber gehörte schon zum antiken Pestbegriff. Zwar wurde im 17.Jahrhundert, als der Bubo schon Bestandteil des Pestbegriffes war, auch über eine Pest ohne Fieber diskutiert ${ }^{16}$; doch ist, davon abgesehen, durch alle Zeiten das Fieber ein Element des Pestbegriffes gewesen.

Das Fieber bildete nun aber nicht nur einen Bestandteil der Pestdefinition, sondern es diente auch dazu, die Pest in das System der galenischen Krankheitslehre einzuordnen, die bekanntlich bis ins 18. Jahrhundert hinein galt. Die Pest wurde nämlich spätestens seit Galen unter dem Oberbegriff der Fieber als pestilenzialisches Fieber klassiert. Dadurch entstand ein Moment der Begriffstrübung in dem Sinne, daß die Pest durch die Einordnung als eines unter zahlreichen Fiebern eine hervorragende Stelle als einheitliche, eigenständige Krankheit nicht einnehmen konnte; die Pest blieb gleichsam in die Fülle der Erscheinungen eingebettet. Für den Laien, der dies nicht im Zusammenhang mit anderen Krank- 
heiten sehen mußte, ein außerordentliches Ereignis, wurde die Pest demnach für den Arzt zu einem Teilelement des Fieberbegriffes.

Um zu zeigen, wie sich das Problem der Pesterkennung bzw. -beschreibung darstellt, wenn von der Fieberlehre ausgegangen wird, sei diese aus Avicenna im Umriß vergegenwärtigt ${ }^{17}$ :

Angelpunkt ist das Fieber, das meist als Krankheit gilt, die, je nachdem sich aus dem Fieber etwas entwickelt oder nicht, verschiedene Erscheinungsweisen hat. Da ist erstens das ephemere Fieber, aus dem sich, wie der Name sagt, nichts entwickelt; es verschwindet, wie es kommt, und hinterläßt eine restitutio ad integrum. Zweitens ist das putride Fieber (Faulfieber) zu erwähnen, bei dem nach der Säftelehre ein Saft oder mehrere Säfte als verdorben angesehen werden, da es schon wesentlich schwerere Veränderungen als das ephemere Fieber hervorruft. Drittens suchen sich gemäß der Säftelehre die verdorbenen Säfte in Form eines Apostemas einen Ausweg an die Körperoberfläche zu verschaffen; solche Fieber sind von Flecken, Papeln, Pusteln oder eben auch von Bubonen und Karbunkeln begleitet und heißen pestilenzialische Fieber. Schließlich gibt es viertens die hektischen Fieber, die sehr schwer verlaufen, weil sie die festen Körperteile angreifen. Diese antik/mittelalterlichen Fieberkategorien lassen in nicht ganz undeutlichen Umrissen - wie wir heute sagen würden - die flüchtigen Viruserkrankungen, die akuten sowie die chronischen Infekte erahnen.

Die Fieberlehre legte auch die Vorstellung nahe, daß ein Fieber sich in ein anderes verwandeln oder sich mit einem anderen kombinieren könne. Aus dem ephemeren kann im Krankheitsablauf ein putrides oder gar ein hektisches werden. Innerhalb der Kategorie des pestilenzialischen Fiebers können aus den Flekken z. B. Pusteln werden usw. Das bedeutet nach den heutigen Begriffen nicht nur, daß sich z. B. bei den Varizellen aus dem initialen Fleck eine Pustel bildet, sondern eben auch, daß sich das Fleckfieber, das im Orte A herrscht, zur Bubonenpest im Orte B verwandeln kann. Die Vorstellung von der Verwandelbarkeit der Fieber wirkte sich hindernd auf die Erfassung und Abklärung der Infektionskrankheiten als eigenständiger Krankheitsbilder aus, fand aber eine gewisse «Bestätigung» in der Realität, weil unter wenig hygienischen Verhältnissen in einem Seuchenzug sehr oft mehrere Infektionskrankheiten gleichzeitig auftreten.

Trotzdem schälten sich allmählich «eigenständige» Fieber aus der Fieberlehre heraus. Anhand der typischen Exantheme wurden zuerst die Pocken und Masern von Rhazes (im 9. Jahrhundert) begrifflich isoliert. Seither stehen in den Büchern «De febribus» die «Varioli» und die «Morbilli» in Einzelkapiteln am Ende der Lehre über die putriden bzw. pestilenzialischen Fieber. Die Pocken und Masern 
sind demnach als eigenständige Krankheitsbilder aufgefallen, indessen nicht die Bubonenpest, die uns so leicht erkennbar scheint.

In welcher Art die Bubonenpest in der Fieberlehre «verborgen» war, soll wiederum am Beispiel Avicennas gezeigt werden. Unter dem Titel «De febre pestilentiali ${ }^{18}$ beschreibt er einen schweren Fieberzustand, der mit Delirien, Magendarmsymptomen, Milzschwellung, vielleicht einem Husten einhergeht; so weit ist sein Pestilenzfieber undifferenziert und unspezifisch. In einem anderen Kapitel ${ }^{19}$ mit dem Titel «Von den heißen und korrupten Apostemen» deutet er an, daß Bubonen bei der Pestilenz und in den Pestgegenden gehäuft auftreten (er unterscheidet ferner noch ein «febris apostematum», das innere, beim Herzen gelegene Aposteme begleitet) ${ }^{20}$. Avicenna hat demnach einerseits deutliche Vorstellungen von schweren Fieberzuständen, die er Pestilenzfieber nennt, und andererseits von Drüsenschwellungen, die mit schweren Fiebern verbunden sind. Aus diesen beiden Elementen findet aber keine eindeutige Synthese zum Bild der Bubonenpest statt, was seinen Grund darin haben mag, daß er diese nur vom Hörensagen her kannte.

Dasselbe gilt mehr oder weniger für die ärztliche Pestvorstellung bis ins 14. Jahrhundert. Dazu ist als Beispiel Bernard de Gordon (Übergang vom 13. zum 14. Jahrhundert) zu erwähnen, der die in Pestzeiten auftretenden Pockenausschläge und Bubonen als schlechtes prognostisches Zeichen wertet ${ }^{21}$. Auch bei ihm besteht kein isoliertes Krankheitsbild der Bubonenpest.

\section{Pest und Apostema}

Das ändert sich beinahe schlagartig mit der Pestbeschreibung von Guy de Chauliac, einem Augenzeugen des vielfach bezeugten Massensterbens von $1347 \mathrm{ff}$. Wenn man davon absieht, daß zur Zeit des Massensterbens im 6. Jahrhundert die damals aufgetretene Krankheit als «morbus inguinarius» usw. bezeichnet wurde - was vielleicht zu Recht als Beulenpest interpretiert worden ist -, so ist Guys Darstellung die erste ärztliche, die das Bild der Bubonenpest deutlicher erfaßt.

«Das besagte Sterben begann bei uns [in Avignon, 1348] im Monat Januar und dauerte sieben Monate lang. Es zeigte sich auf zwei Arten. Die erste dauerte während zweier Monate mit ununterbrochenem Fieber und Blutspeien; man starb innert dreier Tage. Die zweite Art dauerte die ganze übrige Zeit, auch mit ununterbrochenem Fieber und Apostemen und Karbunkeln an den äußeren Teilen, hauptsächlich in den Achselhöhlen und Leistenbeugen; man starb in fünf Tagen. Die Ansteckung war so groß, vor allem die mit Blutspeien, daß man sie nicht nur vom Zusammenleben, sondern auch vom bloßen Anblicken bekam ... Ich nenne es das große Sterben, weil es die ganze Welt befiel, oder wenig hätte dazu gefehlt. Es begann im Osten, kam in unsere Gegend und ging weiter in den Westen. Und es war so groß, daß es kaum ein Viertel der Menschen 
hinterließ. Ich sage, es war so, daß man noch nie von etwas Ähnlichem reden hörte .... ${ }^{22}$

«Daß man noch nie von etwas Ähnlichem reden hörte» weist darauf hin, daß Guy in voller Kenntnis der zu seiner Zeit gängigen medizinischen Literatur diese Pest für etwas wenn nicht Neues, so doch nicht Beschriebenes hielt. Wir gehen deshalb nicht falsch, wenn auch uns Heutigen die verschiedenen Erwähnungen von Beulenkrankheiten im Schrifttum vor dem 14.Jahrhundert schattenhaft erscheinen.

Nicht nur faßte Guy die Bubonen als ein wesentliches Merkmal dieser Seuche auf, sondern er stellte auch die Lungenbeteiligung als zum gleichen Krankheitsbild gehörig dar. Der Pestbegriff wurde demnach durch diese beiden Elemente vervollständigt. Denn daß er diese Seuche trotz ihrer Unerhörtheit und seiner dafür gewählten Bezeichnung «großes Sterben» für eine Pest hielt, geht daraus hervor, daß er für ihr Entstehen die oben vorgestellte Pestzeit verantwortlich machte.

Neu ist indessen, daß Guy die Seuche nicht bei den Fiebern, sondern bei den Apostemen klassiert, was besagt, daß er nicht das Fieber, sondern die Bubonen und Karbunkel als das Charakteristikum ansah. Sowohl die Beschreibung als auch die Klassierung zeigen an, daß er eine Krankheit als eigenständig erkannt hat, die wir heute Bubonenpest nennen. Das soll nicht heißen, daß ihm allein das Verdienst dieser begrifflichen Isolierung zukommt. Man darf vielmehr annehmen, daß diese Pandemie mit signifikanten Mengen gleichartiger Fälle ein derart auffälliges Ereignis war, daß die Eigenständigkeit der Krankheit mit dem Charakteristikum der Beulen vielen gleichzeitig aufgefallen war. So gibt es denn seit 1347 zahlreiche Beschreibungen, die eine retrospektive Diagnose erleichtern.

Es wäre indessen eine Täuschung, wenn man annehmen wollte, daß sich die Auffassung der Bubonenpest als einer eigenständigen Krankheit seither ein für allemal undiskutiert durchgesetzt hat. Die Vorstellung vom pestilenzialischen Fieber hielt sich hartnäckig. Die Auffassung, die Bubonen seien das wesentliche Merkmal der Pest, bewirkte, daß später eine neue Schwierigkeit in der Erfassung des Pestbegriffes entstand. Für Ysbrand van Diemerbroeck (1609-1674), der die Symptome der Bubonenpest - einschließlich des Nasenblutens - sehr wohl kannte, wurde die Pest nun so sehr nur zur Bubonenkrankheit, daß er alle Bubonen, auch diejenigen, die ohne Fieber verlaufen, als zur Pest gehörig betrachtete. Er zog daraus den Schluß, daß zur wahren Pest nicht notwendigerweise auch Fieber gehören müsse. Diese Ansicht begründete er dadurch, daß ein starkes Herz sehr wohl die Abwehrkraft habe, das Pestgift direkt in die Emunktorien zu schicken, ohne dabei im ganzen Körper Fieber zu verursachen ${ }^{23}$. 
Diese an sich vertretbare Idee führte in der diagnostischen Praxis dazu, daß in einer Pestzeit jede Drüsenschwellung als Pest angesehen werden konnte, was sich auch aus van Diemerbroecks eigenen Krankheitsgeschichten bestätigt ${ }^{24}$.

Trug also die Entdeckung, daß der Bubo zur Pest gehört, einerseits dazu bei, diese Krankheit aus dem großen Sammelbecken der Fieber herauszulösen, so hatte dieselbe Entdeckung andererseits zur Folge, daß zahlreiche Krankheiten, die nichts mit der Pest zu tun hatten, dazugerechnet werden konnten.

\section{Pest und Fäulnis}

Gegen das Ende des 16. Jahrhunderts kam die Vorstellung der Fäulnis zum Pestbegriff hinzu. Die Fäulnis, ein leicht ohne technische Hilfsmittel zu beobachtender Zerfallsvorgang, bildete schon in der Antike einen wichtigen Bestandteil der Fieberlehre, der sich in der Idee der putriden Fieber, der zweiten Hauptkategorie der Fieberklassierung, ausdrückte. Fäulnis als Zerfall und fieberhafte Krankheit als Zerfall bildeten zwei Parallelvorstellungen.

Der häufig bei der Fäulnis zu beobachtende Parasitenbefall legte den Gedanken nahe, daß bei der Fieberfäulnis irgendwelche wenn auch unsichtbare Parasiten beteiligt seien. Diese schon antike Idee wurde durch Girolamo Fracastoro im 16. Jahrhundert wieder aufgenommen und in seiner Monographie «De contagione... » breiter als im Altertum im Zusammenhang mit den kontagiösen Krankheiten dargestellt, so daß die gedankliche Verknüpfung Pest - Ansteckung Fäulnis - Parasitenbefall nahegelegt wurde. So spricht z. B. Felix Platter (15361614) vom Pestfieber als einem bösartigen Faulfieber ${ }^{25}$.

Weiter ging der schon erwähnte Athanasius Kircher in seinem «Scrutinium pestis» (1659), das er selbst als «nova doctrina » bezeichnete. Im Rahmen der Pestschriften des 17.Jahrhunderts war es tatsächlich ein Novum, daß er bei seiner Pestbetrachtung nun die größte Emphase eben auf die Vorstellung der Fäulnis und des Parasitenbefalles legte. Er selbst suchte mit dem damals neuen Mikroskop nach solchen Parasiten, die er in vollständiger Analogie zu den am Kadaver zu sehenden Parasiten Würmer nannte. Solche Würmer entstünden ex se aus der Fäulnis und erzeugten die Krankheit Pest ${ }^{26}$. Er glaubte, solche Würmer im Blute Pestkranker erblickt zu haben, was aber wegen des geringen Auflösungsvermögens der damaligen Mikroskope nicht Pesterreger gewesen sein können. Man meint, er habe in Wirklichkeit Erythrozyten gesehen. Seine falsch interpretierte Beobachtung ist indessen das Beispiel einer später als richtig erwiesenen Intuition.

Zwischen Kirchers Intuition und Yersins Entdeckung des Pestbakteriums liegt keine begriffliche, sondern nur eine technische Entwicklung, die allerdings rund 
200 Jahre brauchte. Wir verzichten auf die Nennung der Autoren, die sich mit Kirchers Pestwürmertheorie auseinandergesetzt haben, und verweisen auf die Geschichte der Bakteriologie. Beispielshalber sei Johannes von Muralt genannt, der 1721 dafür hielt, daß «Mücklin die Pestwürmlin ins Blut bringen » ${ }^{27}$. Dies war ein Schritt weiter in der Ahnung der Pathogenese der Pest. Mit der Pestwürmertheorie begann die Vorstellung der Pestzeit allmählich zu verblassen, bis sie mit Yersins Entdeckung aus dem ärztlichen Pestbegriff ausgeschlossen wurde. Doch lebt die Pestzeitvorstellung im 20. Jahrhundert im berühmten Roman von Albert Camus «La peste» gewissermaßen als «Peststimmung» literarisch weiter.

\section{Zusammenfassung}

\section{Pest als Krankheitseinheit}

Ausgehend vom heutigen Pestbegriff, versuchten wir darzulegen, zu welcher Zeit und in welcher Art die Hauptelemente dieses Begriffes sich konstituierten, und wir stellten dabei fest, daß er mit Athanasius Kircher in den Umrissen seine heutige Form fand.

Es ist nun allerdings nicht so, daß mit der Aufnahme der einzelnen Elemente in den Pestbegriff dieser in seiner jeweils ergänzten Form einhellig akzeptiert worden wäre. Vielmehr vertraten die verschiedenen Autoren ihre eigenen, teilweise aus der medizinischen Literatur, teilweise aus der Beobachtung stammenden Ansichten. Insofern ist die dargestellte Übersicht eine Schematisierung. Wir erinnern daran, daß namentlich in der Frage, ob die Pest eine eigenständige Krankheit sei, die Autoren sich nicht oft einig waren.

Wendet man sich zu Guy de Chauliacs Pestbeschreibung zurück, so kann bei ihm von der Pest als spezifischer Krankheitseinheit im eingangs erwähnten Sinne nicht die Rede sein. Obwohl er es nicht ausdrücklich sagt, gewinnt man aber dennoch den Eindruck, daß er die Pest als ein eigenständiges Geschehen sah, als eine Einheit, die mit nichts anderem etwas zu tun hatte.

Joubert spricht von der Pest als von einer Krankheit, deren Fieberelement allerdings zu den übrigen Pestfiebern gehört und als solches zu deren Differentialdiagnose steht ${ }^{28}$. Diese Zweiseitigkeit liegt bei Joubert nicht etwa am mangelnden Realwissen um die Bubonenpest, sondern ist eben durch die begriffliche und terminologische Doppelsinnigkeit gegeben, die ein Erbe der Einbettung der Pest in die Fiebellehre war.

Geronimo Mercuriali wiederum differenziert zehn Jahre nach Joubert zwischen der Pestkrankheit einerseits - er nennt sie die wahre Pest - und den Pestilenzfiebern andererseits - der falschen Pest, die eine Infektionskrankheit der 
Verdauungsorgane gewesen sein dürfte ${ }^{29}$. Die Unterscheidung einer wahren und falschen Pest stellt einen deutlichen Schritt zur Auffassung der Pest als einer Krankheitseinheit dar.

Bei Johann Baptist van Helmont ferner, um 1624, ist die wahre Pest diejenige, die Gott zur Strafe schickt, die falsche dagegen eine solche, die natürliche Ursachen hat. Beide weisen die Symptomatologie der Bubonenpest auf ${ }^{30}$, eine Ansicht, die den diagnostischen Wert der Unterteilung der Pest in eine wahre und falsche illusorisch macht.

Daß Felix Platter die Pest als eingeständige Fieberkrankheit, die Bubonen aber als unbedingt dazugehörige Begleitkrankheit auffaßte, zeigt, daß trotz dem Realwissen $^{31}$ um die Bubonenpest diese gewissermaßen auch für zwei Krankheiten genommen werden konnte ${ }^{32}$.

Kircher muß die Pest als eigenständige Krankheit aufgefaßt haben, was sich aus seinen Bemühungen schließen läßt, einen Pesterreger, d.h. eine mit der Krankheit verbundene Ursache zu finden. Daß die Pest eine spezifische Krankheit ist, legte Yersins Entdeckung nahe.

\section{Pest als Pestzeit-Zeichen}

Parallel zu dieser Entwicklung, die schlußendlich zur Auffassung der Pest als einer spezifischen Krankheit führte, lief bis zur Mitte des 19. Jahrhunderts immer auch die Strömung, welche die Pest nicht als eigenständige Krankheit, sondern als Zeichen einer Seuchenzeit sah, das sich ohne weiteres in ein anderes Zeichen bzw. in eine andere Krankheit verwandeln könne. Wie schon bei der Fieberlehre besprochen wurde, galt es durchaus als möglich, daß aus dem «Zeichen» Fleckfieber das «Zeichen» Pest werden konnte und umgekehrt.

Es wäre allerdings falsch anzunehmen, daß die verschiedenen Exanthemformen, Bubonenkrankheiten usw. aus Mangel an Beobachtungsgabe nicht voneinander unterschieden worden wären. Vielmehr verhielt es sich so, daß die an sich unterschiedlichen erkannten Krankheitserscheinungen in einem Konnex miteinander gesehen wurden, der sie zum Phänomen einer und derselben Pestzeitkrankheit machte.

Wie angedeutet, fand die Vorstellung von der Verwandelbarkeit der Krankheiten in den Polyepidemien ihre Nahrung. Denn es besteht kein Zweifel, daß bei ungünstigen hygienischen Verhältnissen eine Seuche nicht «rein» vorkommt. Demnach wäre es auch heute korrekter, nicht von Pestzügen schlechtweg, sondern von Seuchenzeiten zu reden, in welchen die Bubonenpest vorherrschte. Polyepidemien förderten die Idee der Verwandelbarkeit der Krankheiten und hemmten diejenige der spezifischen Krankheit. In diesem Sinne konnte noch 
Heinrich Haeser in der Mitte des 19. Jahrhunderts sagen, daß sich die Pest aus bösartigen Fiebern entwickle und daß auf der Höhe der Epidemie auch andere Krankheiten den Charakter der Pest annähmen.

«Nicht mit Stricken und Chlorgas bewaffnet wird man diesem Feinde der Türkei entgegen gehen müssen, sondern mit richtigen Ideen über Entwicklungen, Verwandtschaften und natürliche Übergänge der Krankheiten .... ${ }^{33}$

Rund zehn Jahre vor der Entdeckung der tatsächlichen Sachverhalte war es also noch durchaus möglich, solche Vermutungen zu äußern. Sie zeigen, daß es gar nicht so selbstverständlich war, die Pest als eigenständige Krankheit aufzufassen.

\section{Anmerkungen}

${ }^{1}$ Robert Hegglin, Differentialdiagnose der inneren Krankheiten, 11.Aufl., Stuttgart 1969, S. 150.

2 Thomas Sydenham, The Works of Thomas Sydenham. Translated from the Latin edition of Dr. Greenhill by R. G. Latham. Vol. I, London 1848, S. $13 \mathrm{ff}$.

${ }^{3} 1$. Samuel 4 und 5.

${ }^{4}$ Corpus Hippocraticum: Aphorismen IV, 55; Epidemien II und IV.

${ }^{5}$ Galen in den Kommentaren zu den Aphorismen des Hippokrates IV, 35, in den Kommentaren zu den Epidemien des Hippokrates II und in De febribus I.

${ }^{6}$ Euagrius (Scholasticus), Historia ecclesiastica IV, 29.

7 Procopius (Caesarensis), De bello Persico II, 22.

8 Arnold C. Klebs und Karl Sudhoff, Die ersten gedruckten Pestschriften, München 1926.

${ }^{9}$ Georg Sticker, Abhandlungen aus der Seuchengeschichte und Seuchenlehre: 1/1 Die Pest (Geschichte der Pest), Gießen 1908; 1/2 Die Pest (Die Pest als Seuche und Plage), Gießen 1910.

10 Thukydides, Geschichte des Peloponnesischen Krieges. Das zweite Kriegsjahr.

${ }^{11}$ Lazare Rivière, La pratique de médecine avec la théorie, Bd. 2, Lyon 1723, Lib. XVII, sect. 3, S. 793.

${ }^{12}$ Georg Sticker, op. cit. Bd. 1/1, S. 7.

Guy de Chauliac, La Grande Chirurgie, Ausgabe von Edouard Nicaise, Paris 1890, S. 167-174.

${ }^{13}$ Georg Sticker, op. cit. Bd. 1/2, S. 167.

${ }^{14}$ Georg Sticker, Über den Ursprung und die Verbreitungsmittel der Pest. In: Hochland 1, München 1904, und op. cit. Bd. 1/2, S. $137 \mathrm{ff}$.

${ }^{15}$ Laurent Joubert, De peste liber unus, in: Jouberti Opera Latina, Lyon 1582, tom. 1, cap. 1 und 2.

${ }^{16}$ Ysbrand van Diemerbroeck, De peste, Amsterdam 1665, hist. IX.

Lazare Rivière, op. cit. Lib. XVII, Kap. 1, S. 795.

${ }^{17}$ Avicenna, Canon medicinae, Basel 1556, Lib. IV, fen I, De febribus, tract. I-IV.

18 ibid. fen 1 , tract. 4 , cap. 1 .

19 ibid. fen 3 , tract. 1 , cap. 17.

20 ibid. fen 1, tract. 4, cap. 13. 
${ }^{21}$ Bernard de Gordon, Lilium medicinae, Lyon 1574, part. I, cap. 10.

${ }^{22}$ Guy de Chauliac, loc. cit.

${ }^{23}$ Ysbrand van Diemerbroeck, loc. cit. Annotatio.

24 ibid.

${ }^{25}$ Felix Platter, Praxeos, 3. Aufl., Basel 1656, Bd. 2, Spalte 12.

${ }^{26}$ Athanasius Kircher, Scrutinium pestis, Leipzig 1659, Kap. 7.

${ }^{27}$ Johannes von Muralt, Kurtze und Grundliche Beschreibung der ansteckenden Seuche der Pest, Zürich 1721, zitiert in: Georg Sticker, op. cit. sub 9, Bd. 1/2, S. $14 \mathrm{ff}$.

${ }^{28}$ Laurent Joubert, op. cit. cap. 6.

${ }^{29}$ Geronimo Mercuriali, Medicina practica, Lyon 1623, Lib. V, cap. 17.

${ }^{30}$ Johannes Baptista van Helmont, Opera omnia: Tumulus pestis, Frankfurt 1707, zitiert in: Georg Sticker, op. cit. sub 9, Bd. 1/2, S. 8.

${ }^{31}$ Huldrych M. Koelbing, Diagnose und Ätiologie der Pest bei Felix Platter (1536-1614), in: Medizinische Diagnostik in Geschichte und Gegenwart, Festschrift für Heinz Goerke zum sechzigsten Geburtstag, München 1978, S. 217-226.

${ }^{32}$ Felix Platter, loc. cit.

${ }^{33}$ Heinrich Haeser, Lehrbuch der Geschichte der Medicin und der Volkskrankheiten, Jena 1845 (3. Auflage in 3 Bänden 1875-1882), zitiert in Georg Sticker, op. cit. sub 9, Bd.1/2, S. 35 .

\section{Summary}

A retrospective diagnosis is not a certain, but a probable one, because its criteria are based on symptomatology only which in ancient authors varies in exactness. This is also the case with plague.

The original meaning of plague is a very deadly mass disease. Already in antiquity, the notion of contagion came to be added to the concept of plague, whereby the modus of contagion remained open to speculation until the late 19th century. Fever also belongs to the ancient concept of plague and was furthermore used to classify plague as a «febris pestilentialis». The pathognomonic bubo enters plague descriptions in the time of the Black Death in the 14th century, viz. the description by Guy de Chauliac. There is, however, a shadowy mentioning of bubones during the epidemic in the time of the emperor Justinianus. The notion of parasitic putridity was taken up by Athanasius Kircher and foreshadows the discovery of the Pasteurella pestis by Yersin and Kitasato in 1894.

There was much controversy amongst medical authors, whether plague was a specific disease entity or merely the most terrible manifestation of a fever and as such capable of metamorphosis. Only as a sequel of Yersin's discovery do we have a clear concept of plague.

Frau Dr.med.Antoinette Stettler

Hallwylstraße 24

3005 Bern 